Original article

\title{
The utility of outpatient commitment: Reduced-risks of victimization and crime perpetration
}

\author{
Steven P. Segal ${ }^{a, *}$, Lachlan Rimes $^{b}$, Stephania L. Hayes ${ }^{c}$ \\ a University of California, Berkeley, U.S.A. E University of Melbourne, Australia \\ ${ }^{\mathrm{b}}$ Victorian Department of Health and Human Services, Australia \\ ${ }^{\mathrm{c}}$ University of California, Berkeley, U.S.A.
}

\section{A R T I C L E I N F O}

\section{Article history:}

Received 11 September 2018

Received in revised form 6 December 2018

Accepted 8 December 2018

Available online 14 January 2019

\section{Keywords:}

Outpatient civil commitment

Community treatment orders

Forensic psychiatry

\begin{abstract}
A B S T R A C T
Background: Outpatient civil commitment (OCC) provisions, community treatment orders (CTOs) in Australia and Commonwealth nations, are part of mental health law worldwide.

This study considers whether and by what means OCC provides statutorily required "needed-treatment" addressing two aspects of its legal mandate to protect the safety of self (exclusive of deliberate-self-harm) and others.

Method: Over a 12.4-year period, records of hospitalized-psychiatric-patients, 11,424 with CTOassignment and 16,161 without CTO-assignment were linked to police-records. Imminent-safety-threats included perpetrations and victimizations by homicides, rapes, assaults/abductions, and robberies. "Need for treatment" determinations were validated independently by Health of the Nations Scale (HoNOS) severity-score-profiles. Logistic regressions, with propensity-score- adjustment and control for 46 potential confounding-factors, were used to evaluate the association of CTO-assignment with occurrence-risk of perpetrations and victimizations.

Results: CTO-assignment was associated with reduced safety-risk: $17 \%$ in initial-perpetrations, $11 \%$ in initialvictimizations, and $22 \%$ for repeat-perpetrations. Each ten-community-treatment-days in interaction with CTO-assignment was associated with a 3.4\% reduced-perpetration-risk. CTO-initiated-re-hospitalization was associated with a $13 \%$ reduced-initial-perpetration-risk, a 17\% reduced-initial-victimization-risk, and a $22 \%$ reduced-repeat-victimization-risk. All risk-estimates appear to be the unique contributions of the CTO, CTO-initiated-re-hospitalization, or the provision of ten-community-treatment-days-i.e. after accounting for the influence of prior crimes and victimizations, ethnic-bias, neighborhood disadvantage and other between-group differences in the analysis.

Conclusions: CTO assignment's association with reduced criminal-victimization and perpetration-risk, in conjunction with requiring participation in needed-treatment via re-hospitalization and communityservice, adds support to the conclusion that OCC is to some extent fulfilling its legal objectives related to protecting safety of self (exclusive of deliberate-self-harm), and others.
\end{abstract}

(c) 2018 Elsevier Masson SAS. All rights reserved.

\section{Introduction}

The need for treatment to protect health and safety is the key provision justifying the use of outpatient civil commitment (OCC), also known as a community treatment order (CTO) under Australian law and in commonwealth countries. OCC-statutes, though varied by jurisdiction in interpretation and language,

\footnotetext{
* Corresponding author at: Honorary Professor University of Melbourne, Australia; and Professor of the Graduate Division and Director, Mental Health and Social Welfare Research Group, School of Social Welfare, 120 Haviland Hall (MC\#7400), University for California, Berkeley, CA, 94720-7400, U.S.A.

E-mail address: spsegal@berkeley.edu (S.P. Segal).
}

almost universally provide for protecting safety from actions associated with behavior considered an imminent danger or harm to self and others [1-4]. The U.K. Parliament's intention for the use of the CTO in the U.K. Mental Health Act of 2007 was to " . . put [the assignment to a CTO] to the clinical decision about the risk in the community .... [5]." This study investigates the potential of CTO-assignment for people with severe mental illness (SMI) to fulfill two aspects of its legal mandate to provide needed treatment to "protect the safety of self and others"-i.e. protection of self from external-threats, victimization (exclusive of deliberate-self-harm), and protection of others from perpetrated-crimes.

A small proportion of individuals with SMI commit major crimes (i.e. homicides, rapes, assaults/abductions, and robberies). Yet, the risk of perpetrating such crimes in the SMI population, 
especially for those with untreated mental illness, has been found to be elevated by approximately three to 13 times when compared to general population-samples internationally in repeated studies [6]. Of equal concern for people with SMI is the population's elevated-risk of victimization by crime. Adjudicated "helpless to avoid the hazards of freedom .... .mentally ill persons who meet this standard are clearly dangerous to themselves [2]." Depending on the type of violent crime, victimization-prevalence-rates are reported to be 6-23 times greater among persons with SMI than among the general population [7]. Teplin [7], p.2] notes that: "symptoms associated with SMI, such as impaired reality testing, disorganized thought processes, impulsivity, and poor planning and problem solving, can compromise one's ability to perceive risks and protect oneself [8-13]. Moreover, factors correlated with victimization-substance abuse, conflicted social relationships, poverty, and homelessness [14-17]-are common among persons with SMI $[8,18,19] . "$

Previous individual-focused-research has reported associations of OCC with reduced safety-risks. These studies found OCC associated with reduced victimization (self-reports of crimes against persons and property) [20], arrests of all types [21], violent incidents, [22], and reduced mortality-risk [23-25].

In Victoria, Australia, the CTO-statute is characteristic of most international jurisdictions (see, Appendix, Section I). The primary criteria for CTO-assignment involves an assessment indicating that the patient, due to mental illness, is in need of treatment to protect health and safety. The patient must be refusing such treatment and the treatment must be the least restrictive form of care. The patient can only be placed and/or retained on a CTO for the period her/his behavior continues to be a near-term threat to health and safety and during which treatment is available. The CTO-supervisedtreatment functions as "a less restrictive alternative to psychiatric hospitalization" [26] by releasing inpatients whose behavior continues to pose health and safety risks earlier than would typically occur or by allowing continued community residence of patients posing health and safety risks without initial hospitalization. Both actions enable reduced inpatient time and potentially reduce community-risk [27].

The CTO requires an individual to participate in communitybased mental health treatment believed to reduce their risk of involvement in actions that are harmful. CTO-supervision also enables a timely return to an inpatient facility for treatment when new symptoms threaten to have dangerous or harmful consequences, when patients fail to comply with CTO-treatmentagreements, and when community care is either unavailable or ineffective (a situation where no less restrictive alternative exists). When the CTO is not complied with or is deemed ineffective-i.e. the patient is refusing required treatment, deemed "needed", and their mental health is deteriorating-they can, in all Australian jurisdictions and most international ones, be directly returned to a psychiatric hospital [1,4]. There it is determined if they continue to manifest the potentially harmful behavior that originally brought them into involuntary care and require readmission $[1,4]$. Previous population-focused-research has reported an association between reduced homicide rates and the use of the "need for treatment" criterion in civil commitment, in both inpatient and outpatient settings [28]. Bringing people to hospital earlier in the course of their first psychotic episode for needed-treatment was found to be associated with reduced homicide rates [29]. Using OCC to bring people in earlier to hospital in recurrent episodes of illness may have a similar outcome.

All Victoria's psychiatric hospitals were closed in 2000 and the primary state-governmental-unit responsible for communitybased-care was disbanded [30]. This unit had built a rather comprehensive community-care-system during the decade prior to this investigation, a system designed to ensure service for a deinstitutionalized population in community-based specialtymental health services [30]. With the demise of this unit, and a shift to integrated-care focused around the general hospital, Victoria experienced a $25 \%$ reduction in community-based service, noted by comparing its offerings from 1990 to 2000 with those from 2000 to 2010 [27]. This reduction may have refocused the function of the CTO to crisis-management, given that $39 \%$ of CTOs ended in re-hospitalization [27]

This study focuses on questions of safety (exclusive of deliberate self-harm) in Victoria, Australia. It considers whether CTO-supervision is associated with reducing occurrence-risk of two major indicators of imminent danger or harm to self or others-i.e. perpetration and victimization of major crimes against persons. It also considers whether community-treatment-provision and the provision of needed treatment via timely return to an inpatient-facility while under CTO-supervision is associated with reduced-risks of dangerous or harmful behavior. Given the statutory mandates in the law with respect to the use of CTOs, it is therefore hypothesized that the experience of a CTO: 1) Will be associated with a reduced-risk of perpetrating and/or being a victim of "a major-crime-against-a-person". 2) Will be associated with a reduced-risk of repeated major-crime-experience. And, 3) that providing community-based-treatment and/or employing CTO-supervision to return a person to the hospital will be associated with a reduced-risk of initial and repeat perpetrations and victimizations.

\section{Method}

Patient records from Victoria, Australia, were obtained from the Victorian Psychiatric Case Register/RAPID system for all 11,424 who experienced psychiatric hospitalization and/or their first CTO between 2000 and 2010, and 16,161 (matched and randomly selected patients) who had experienced psychiatric hospitalization without CTO-assignment (see Appendix, Section II A, for sampling details). Mental health records were linked via the Victoria Police Law Enforcement Assistance Program (LEAP) [31] to all police contacts in the State extending for an additional 2.4-year-period, yielding a 12.4-year study-period. Additionally, they were linked to records of: Corrections Victoria (documenting detention in police custody or prison), the Socio-Economic Indexes for Areas (SEIFA) (documenting neighborhood disadvantage [32]), and the Australian Mental Health Outcomes and Classification Network's (AMHOCN) Health of the Nation Outcome Scale (HoNOS) assessments of symptom-severity-profiles (documenting potential need for treatment) [33]).

\subsection{Measurement}

Mental health service/treatment contacts. A service contact in Victoria entails medication, supervision, therapeutic discussion, and support in daily activities. It “ . . . is clinically significant in nature [and] includes activity which directly contributes towards . . . the therapeutic needs of a client's condition." [34] (See: Appendix, Section II.B.2).

Mental health episodes of care. All mental health treatment contacts (inpatient, voluntary outpatient community care, and CTO) were organized into episodes of care [35]. Each psychiatric hospitalization (from day of admission to discharge) was considered a separate inpatient-episode. Each continuous period of outpatient-care without a break in service for 90 days or more defined a community-care episode [35]. Service-breaks of 90 days or more followed by re-initiation of care defined the start of a new community-care episode. Each CTO-episode began when a patient was placed on orders and ended when the order was terminated. A community-treatment-day was any day during a community-care 
episode (voluntary or CTO) when the patient received at least one mental health system service contact.

Measures of imminent threats to safety were limited to LEAPreports of perpetrations of and victimizations by major crimes against persons, i.e. homicides, rapes, assaults/abductions, and robberies [36]. Victoria's crime incident rates were computed using LEAP data from 2000 to 2012 [31], the 12.4-year study period, and Victoria's Australian Bureau of Statistics population data [37]. Incident rates were computed for each year and the mean of the rates over the 12.4-year period was reported herein.

Measuring Neighborhood Disadvantage. All the postal-codes in Victoria are SEIFA-ranked for socio-economic disadvantage, ranging from one (most disadvantaged) to 644 [32]. The lowest ranked postal-code where the individual lived during the study was used as the indicator of their neighborhood socio-economic disadvantage [38].

Validating and accounting for a need for treatment. Clinician determinations of a need for treatment at inpatient admission and discharge (when CTO-placement typically occurred) were validated with independent Health of the Nations Scale (HoNOS) severityscore profile assessments conducted in parallel to the CTOevaluations [27]. The Health of the Nation Outcome Scale (HoNOS) is a 12-dimension-profile with established reliability and validity [33]. Individual HoNOS dimensions are rated from zero, no problem, to four, an extremely problematic situation [39]. A serious problem with any HoNOS-dimension would contribute to a decision of eligibility for CTO-placement validating a need for treatment determination [40-44].

\subsection{Analyses}

Study analyses used SPSS 24.0 [45]. Logistic regression models with propensity-score adjustment included the following variables to address study hypotheses.

Outcome variables: "having committed at least one perpetration", "being a victim of at least one victimization", and engaging in a "repeat perpetration" or a "repeat victimization" (CTO's potential associations with recidivism) were each coded 1=presence, $0=$ absence.
Intervention-effect-measures as independent variables. All models were run twice, once with "CTO-cohort membership" ( 1 = CTO vs $0=$ non-CTO) as the intervention-effect independent variable and once with "CTO-initiated re-hospitalization" as the interventioneffect independent variable. All models initially included a measure of community-treatment-days, and subsequently the number of units of "ten community-treatment-days" the patient experienced in a community-care episode and an evaluation of observed significant interaction effects between "ten communitytreatment-days" and CTO-assignment. Ten-community-treatment-days per episode was chosen because it was the average difference between the numbers of community-treatment-days provided to the two cohorts. The "number of communitytreatment-days per month" and the "number of communitytreatment-hours per treatment day" were considered measures of treatment intensity and were evaluated in the models.

Confounding influences as independent variables: In addition to the intervention measures, a propensity-score adjusting for selection into the CTO-cohort, and involvement in crime/victimization prior to the study, the following potential confounding influences were entered into the models stepwise in three blocks:

Block 1: SEIFA ranking [32], potential bias-indicators (NonEnglish Speaker, Aboriginal and/or Torres Strait Islander status), and imprisonment or held in custody during the study period.

Block 2: Demographic, diagnostic, risk-period, and service use indicators including: gender, age, unemployment status, age at entry to the mental health system, time in the study, diagnosis (schizophrenia, affective disorder, dementia, paranoia), duration of inpatient care, episode start year, and $<11$ th grade education,

Block 3: Need for treatment validity controls: Twenty-four HoNOS-items assessing the severity of symptom profiles, 12 measured at inpatient admission, and 12 at hospital-release [39].

Additional detailing of the model theory and the basis of variable selection is available online (see: Appendix, Section-II-B, C \& D).

Challenges to generalizability. OCC/CTO statutes-in the U.S., U.K., Australia, Norway, as well as other jurisdictions address risks attributable to "mental illness" without diagnostic restrictions $[1,4]$. Yet for purposes of comparison with other studies, final

Table 1

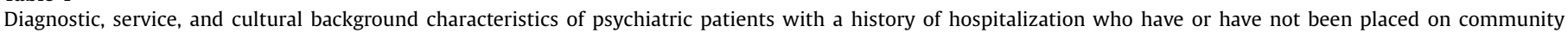
treatment orders.

\begin{tabular}{|c|c|c|c|c|c|c|c|c|c|}
\hline \multirow[b]{2}{*}{ Characteristic } & \multicolumn{3}{|c|}{$\begin{array}{l}\text { Patients with history } \\
\text { of hospitalization }(N=27,585)^{*}\end{array}$} & \multicolumn{3}{|c|}{ CTO Cohort $(\mathrm{N}=11,424)^{*}$} & \multicolumn{3}{|c|}{ Non-CTO Cohort $(\mathrm{N}=16,161)^{*}$} \\
\hline & $\mathrm{N} / \mathrm{M} \pm \mathrm{sd}$ & $\%$ & $\begin{array}{l}\text { Median/ } \\
\text { Range/ } \\
\text { Interquartile } \\
\text { Range }\end{array}$ & $\mathrm{N} / \mathrm{M} \pm \mathrm{sd}$ & $\%$ & $\begin{array}{l}\text { Median/ } \\
\text { Range/ } \\
\text { Interquartile } \\
\text { Range }\end{array}$ & $\mathrm{N} / \mathrm{M} \pm \mathrm{sd}$ & $\%$ & $\begin{array}{l}\text { Median/ } \\
\text { Range/ } \\
\text { Interquartile } \\
\text { Range }\end{array}$ \\
\hline \multicolumn{10}{|l|}{ Gender } \\
\hline Male & 15,480 & 56 & & 6,646 & 58 & & 8,834 & 55 & \\
\hline Female & 12,103 & 44 & & 4,776 & 42 & & 7327 & 45 & \\
\hline Age at entry to mental health system & $34.0 \pm 16.7$ & & & $32.0 \pm 15.3$ & & & $35.4 \pm 17.5$ & & \\
\hline Never married & 13,410 & 49 & & 6,005 & 53 & & 7,405 & 46 & \\
\hline \multicolumn{10}{|l|}{ Diagnosis } \\
\hline Schizophrenia disorder & 18,260 & 66 & & 8,240 & 72 & & 10,020 & 62 & \\
\hline Major affective disorder & 2,760 & 10 & & 920 & 8 & & 1,840 & 11 & \\
\hline Paranoia or psychotic disorders & 2,157 & 8 & & 719 & 6 & & 1,438 & 9 & \\
\hline Dementia or other nervous system disorder & 3,167 & 11 & & 1,131 & 10 & & 2,036 & 14 & \\
\hline Other disorder & 912 & 3 & & 305 & 4 & & 607 & 4 & \\
\hline Lifetime inpatient days & $86 \pm 247$ & & $24 / 5478 / 64$ & $151 \pm 313$ & & $60 / 5478 / 105$ & $40 \pm 171$ & & $8 / 4016 / 28$ \\
\hline Community treatment episodes & $4.4 \pm 3.9$ & & $3.0 / 65 / 4$ & $6.0 \pm 4.4$ & & $5.0 / 65 / 5$ & $3.3 \pm 2.9$ & & $2.0 / 38 / 3$ \\
\hline Community treatment days per community care episode ${ }^{* *}$ & $20.5 \pm 28.6$ & & $12.7 / 896 / 21$ & $26.6 \pm 30.1$ & & $19.0 / 896 / 22$ & $16.1 \pm 26.7$ & & $8.3 / 824 / 16$ \\
\hline Community treatment days per month** & $8.2 \pm 9.9$ & & $3.0 / 29 / 11$ & $12.5 \pm 11.4$ & & $7.9 / 29 / 23$ & $5.4 \pm 7.6$ & & $1.8 / 29 / 5$ \\
\hline \multicolumn{10}{|l|}{ Socioeconomic Status } \\
\hline Unemployed & 16,604 & 60 & & 7,817 & 69 & & 8,787 & 54 & \\
\hline$<11^{\text {th }}$ Grade Education & 8,806 & 32 & & 3,731 & 32 & & 5,075 & 31 & \\
\hline Lowest SEIFA rand of neighborhood residence & $250 \pm 194$ & & $219 / 643 / 355$ & $230 \pm 191$ & & $190 / 643 / 343$ & $265 \pm 195$ & & $233 / 643 / 362$ \\
\hline
\end{tabular}


models were re-run excluding patients with dementia and other nervous system disorders.

\subsection{Ethics}

Study procedures were approved by ethics committees of the Victorian Department of Health and Human Services, the University of California, Berkeley, and the Victoria Police. Authors have no conflicts of interest to report.

\section{Results}

The average age of the sample $(\mathrm{N}=27,585)$ was $39 \pm 17.3,56 \%$ were males, $31.9 \%$ had $<12^{\text {th }}$ grade education, $60 \%$ were unemployed, and $49 \%$ had never been married. Their diagnoses were schizophrenia (66\%), major affective disorder (10\%), paranoid or psychotic disorder (8\%), dementia and other nervous system disorders (11\%), and other disorders (3\%) (Table 1).

During the study period, the sample logged 14,774 perpetrations, including 140 homicides, 886 rapes, 12,235 assaults and abductions, and 1513 robberies. They suffered an aggregate of 6991 victimizations, including 23 homicides, 1073 rapes, 5293 assaults or abductions and 602 robberies (Table 2).

The CTO-cohort had almost twice the number of communitytreatment episodes $(6.0 \pm 4.4$ vs. $3.3 \pm 2.9)$ than the non-CTOgroup, with almost $40 \%$ more treatment-days per episode $(26.6 \pm 30.1$ vs. $16.1 \pm 26.7)$. Of their community-treatment-episodes, $2.3 \pm 2.4$ involved a CTO ( $46 \%$ had $\geq 2$ CTO-episodes). Overall, the CTO-cohort experienced 25,696 CTO-episodes whose duration was $M=220.7 \pm 256.5$ days (Median=158.1; Interquartile range 269 days). Of these, 5.9\% were initiated from the community, and $39.2 \%$ ended in re-hospitalization (see Table 1 ).

Based upon group characteristics (Table 2), crude perpetration rates per 100,000 during the study were respectively for the CTO and Non-CTO cohorts, 7.5 and 5.6-times higher than for Victoria's citizens. Crude victimization rates were respectively 3.7 and 3times higher. During the study period, the CTO-cohort members logged 7182 perpetrations and 3251 victimizations compared to 7592 perpetrations and 3740 victimizations for the Non-CTOcohort. The CTO- cohort had a significantly higher number of perpetrations and victimizations per person than the Non-CTOcohort: respectively, $0.63 \pm 2.2$ versus $.47 \pm 2.0$ perpetrations per person $(\mathrm{F}=38.08$, df $1,27,584, \mathrm{p}<.001)$ and $.29 \pm .8$ versus $.23 \pm .82$ victimizations per person $(\mathrm{F}=27.39$, df $1,27,584, \mathrm{p}<.001)$.

Focusing on individual involvement in at least one perpetration and/or victimization (Table 3), a greater proportion of the CTO than the non-CTO-cohort had committed at least one perpetration (17\% vs $13 \%$ ) and had been the victim of at least one victimization (16\% vs $13 \%$ ). These perpetration and victimization figures however do not take into account the between-group differences that affect risk differentially in the two cohorts.

Most notably the Non-CTO-cohort lived in better neighborhoods (SEIFA Ranking, 250 vs 219, $\mathrm{F}=216.61$, $\mathrm{df}=1,27,175, \mathrm{p}<.001$ ) and experienced less unemployment (54\% vs $69 \%$, ChiSq $=628.56$, $\mathrm{df}=1, \mathrm{p}<.001$ ) both factors associated with less crime-risk. Additionally, regardless of cohort, perpetrators and victims lived in more disadvantaged neighborhoods. Their neighborhoods differed from those without recorded incidents by ten SEIFApercentile-ranks (28th vs. 38th percentile for the CTO-cohort and 33rd vs. 43rd percentile for the non-CTO-cohort; see Table 3).

Table 2

Exposure to CTOs, crime and victimization incidents over 12.4 years among psychiatric patients with a history of hospitalization ${ }^{\mathrm{a}}$.

\begin{tabular}{|c|c|c|c|c|c|c|c|c|c|c|}
\hline \multicolumn{11}{|l|}{ Victoria's Rate } \\
\hline \multirow[b]{2}{*}{$\begin{array}{l}\text { Incident Specific } \\
\text { Category: }\end{array}$} & \multirow{2}{*}{$\begin{array}{l}\text { Total } \\
\text { Sample } \\
\mathrm{N}=27,585 \\
\text { Total } \\
\text { Sample's } \\
\text { \# of crime } \\
\text { incidents } \\
\text { in } \\
12.4 \text { years }\end{array}$} & \multicolumn{4}{|c|}{ Incidents Attributable to CTO Cohort $\mathrm{N}=11,424$} & \multicolumn{4}{|c|}{ Incidents Attributable to Non-CTO Cohort N=16,161 } & \multirow{2}{*}{$\begin{array}{l}\text { Victoria's Average } \\
\text { Yearly Incident } \\
\text { Rate Per } 100,000 \\
\text { for study years }\end{array}$} \\
\hline & & $\begin{array}{l}\text { \# of incidents } \\
\text { attributed to } \\
\text { CTO Cohort in } \\
12.4 \text { years }\end{array}$ & $\begin{array}{l}\text { Rate per } \\
100,000 \\
\text { for } 12.4 \text { year } \\
\text { study } \\
\text { period }^{\text {b }}\end{array}$ & $\begin{array}{l}\begin{array}{l}\text { Average } \\
\text { yearly rate } \\
\text { per }\end{array} \\
100,000 \text {, } \\
\text { CTO Cohort }\end{array}$ & $\begin{array}{l}\text { CTO to } \\
\text { Victoria } \\
\text { Risk } \\
\text { Ratio }^{\text {d }}\end{array}$ & $\begin{array}{l}\text { \# of incidents } \\
\text { attributed to } \\
\text { Non-CTO } \\
\text { Cohort in } \\
12.4 \text { years }\end{array}$ & $\begin{array}{l}\text { Rate per } \\
100,000 \text { per } \\
12.4 \text { year } \\
\text { study } \\
\text { period }^{\text {b }}\end{array}$ & $\begin{array}{l}\text { Average } \\
\text { yearly rate } \\
\text { for } 100,000 \text {, } \\
\text { non-CTO } \\
\text { Cohort }^{\mathrm{c}}\end{array}$ & $\begin{array}{l}\text { Non- } \\
\text { CTO to } \\
\text { Victoria } \\
\text { Risk } \\
\text { Ratio }^{\text {d }}\end{array}$ & \\
\hline Column ID & A & B & $\mathrm{C}$ & $\mathrm{D}$ & $\mathrm{E}$ & $\mathrm{F}$ & G & $\mathrm{H}$ & I & $\mathrm{J}$ \\
\hline 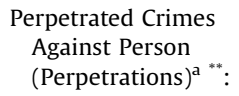 & 14,774 & 7182 & 62,897 & 5072.3 & 7.5 & 7592 & 46,977 & 3788.5 & 5.6 & 680.7 \\
\hline Homicide & 140 & 51 & 446 & 36.0 & 9.7 & 89 & 550.7 & 44.4 & 12.0 & 3.7 \\
\hline Rape & 886 & 414 & 3,624 & 292.2 & 10.0 & 472 & 2,921 & 235.5 & 8 & 29.3 \\
\hline $\begin{array}{l}\text { Assault/ } \\
\text { Abduction** }\end{array}$ & 12,235 & 6,003 & 52,547 & 4237.7 & 7.2 & 6,232 & 38,561 & 3109.8 & 5.3 & 586.4 \\
\hline Robbery & 1513 & 714 & 6,250 & 504.0 & 8.2 & 799 & 4,944 & 398.7 & 6.5 & 61.3 \\
\hline $\begin{array}{l}\text { Victimizations by } \\
\text { Crimes Against } \\
\text { Persons ( } \\
\text { Victimizations) }{ }^{a^{* *}} \text { : }\end{array}$ & 6991 & 3251 & 28,458 & 2295.0 & 3.7 & 3740 & 23,142 & $1,866.3$ & 3 & 612.2 \\
\hline Homicide & 23 & 5 & 44 & 3.5 & 1.0 & 18 & 111 & 9.0 & 2.5 & 3.6 \\
\hline Rape $^{*}$ & 1073 & 515 & 4,508 & 363.5 & 15.5 & 558 & 3,452 & 278.4 & 11.9 & 23.4 \\
\hline $\begin{array}{l}\text { Assault/ } \\
\text { Abduction** }\end{array}$ & 5293 & 2,444 & 21,393 & 1725.2 & 3.2 & 2,849 & 17,629 & 1421.37 & 2.7 & 530.8 \\
\hline Robbery $^{*}$ & 602 & 287 & 2,512 & 202.6 & 3.7 & 315 & 1,949 & 157.2 & 2.9 & 54.3 \\
\hline
\end{tabular}

${ }^{a}$ Crimes and victimizations against persons (CAPs \& VAPs) include all homicides, rapes, assaults/abductions, and robberies.

${ }^{\mathrm{b}}$ The rate per $100,000=\left[\left(\mathrm{B} / \mathrm{N}_{\mathrm{CTO}}\right)(100,000)\right]$ or $\left[\left(\mathrm{F} / \mathrm{N}_{\text {noncto }}\right)(100,000)\right]$.

${ }^{c}$ verage Yearly Incident Rate Per 100,000 for the study years= C/12.4 for CTO cohort; G/12.4 for non-CTO cohort.

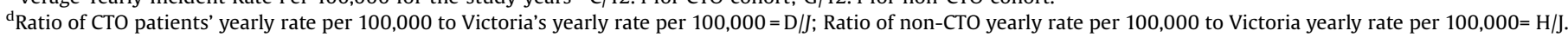

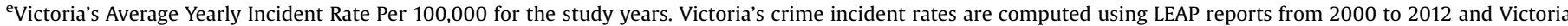
population data. The crime incident rate was computed for each year. The mean of the rates taken over the 12.4-year period is reported herein.

${ }^{*}$ CTO and non-CTO samples differed significantly at between $\mathrm{p}=.001$ and $\mathrm{p}=.005$.

** CTO and non-CTO samples differed significantly at $\mathrm{p}<.001$. 
Table 3

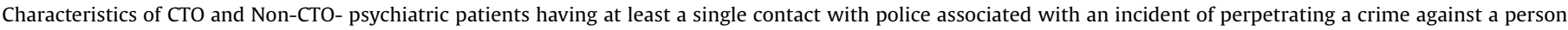
(Perpetration) or being a victim of a crime against person (Victimization).

\begin{tabular}{|c|c|c|c|c|c|c|c|c|c|c|c|c|c|c|c|c|}
\hline \multirow{2}{*}{$\begin{array}{l}\text { Cohort Membership } \\
\text { Perpetrated or was a victim of at least one crime } \\
\text { against person }\end{array}$} & \multicolumn{8}{|c|}{$\begin{array}{l}\text { CTO Cohort } \\
\mathrm{N}=11,424(100 \%)\end{array}$} & \multicolumn{8}{|c|}{$\begin{array}{l}\text { Non-CTO Cohort } \\
\mathrm{N}=16,161(100 \%)\end{array}$} \\
\hline & \multicolumn{2}{|c|}{$\begin{array}{l}\text { Perpetration } \\
\mathrm{n}=1998 \\
(17 \%)\end{array}$} & \multicolumn{2}{|c|}{$\begin{array}{l}\text { No } \\
\text { Perpetration } \\
\mathrm{n}=9426 \\
(83 \%)\end{array}$} & \multicolumn{2}{|c|}{$\begin{array}{l}\text { Victimization } \\
\mathrm{n}=1885 \\
(16 \%)\end{array}$} & \multicolumn{2}{|c|}{$\begin{array}{l}\text { No } \\
\text { Victimization } \\
\mathrm{n}=9540 \\
(84 \%)\end{array}$} & \multicolumn{2}{|c|}{$\begin{array}{l}\text { Perpetration } \\
n=2131 \\
(13 \%)\end{array}$} & \multicolumn{2}{|c|}{$\begin{array}{l}\text { No } \\
\text { Perpetration } \\
\mathrm{n}=14,030 \\
(87 \%)\end{array}$} & \multicolumn{2}{|c|}{$\begin{array}{l}\text { Victimization } \\
\mathrm{n}=2110 \\
(13 \%)\end{array}$} & \multicolumn{2}{|c|}{$\begin{array}{l}\text { No } \\
\text { Victimization } \\
n=14,051 \\
(87 \%)\end{array}$} \\
\hline \multicolumn{17}{|l|}{ Characteristic } \\
\hline Statistics & $M$ & $S D$ & $M$ & $S D$ & $M$ & $S D$ & $M$ & $S D$ & $M$ & $S D$ & $M$ & $S D$ & $M$ & $S D$ & $M$ & $S D$ \\
\hline Age $(\mathrm{M} \pm \mathrm{SD})$ at mid-study & 30 & 10 & 40 & 17 & 31 & 11 & 40 & 17 & 31 & 10 & 41 & 18 & 31 & 11 & 41 & 18 \\
\hline SEIFA Score ${ }^{*}$ & 180 & 173 & 240 & 194 & 180 & 175 & 240 & 193 & 206 & 179 & 274 & 195 & 215 & 182 & 272 & 195 \\
\hline Statistics & $N$ & $\%$ & $N$ & $\%$ & $N$ & $\%$ & $N$ & $\%$ & $N$ & $\%$ & $N$ & $\%$ & $N$ & $\%$ & $N$ & $\%$ \\
\hline \multicolumn{17}{|l|}{ Gender: } \\
\hline Male & 1,567 & 78 & 5,079 & 54 & 1,093 & 58 & 5,553 & 58 & 1,726 & 81 & 7,616 & 54 & 1,218 & 58 & 7,616 & 54 \\
\hline Female & 431 & 22 & 4,345 & 46 & 791 & 42 & 3,985 & 42 & 405 & 19 & 6,435 & 46 & 892 & 42 & 6,435 & 46 \\
\hline \multicolumn{17}{|l|}{ Education: } \\
\hline$<11$ th grade & 823 & 41 & 2,908 & 31 & 744 & 39 & 2,987 & 31 & 969 & 45 & 4,106 & 29 & 859 & 41 & 4,216 & 30 \\
\hline Other & 1,175 & 59 & 6,518 & 69 & 1,141 & 61 & 6,552 & 69 & 1,162 & 55 & 9,924 & 71 & 1,251 & 59 & 9,835 & 70 \\
\hline \multicolumn{17}{|l|}{ Employment: } \\
\hline Unemployed & 1,536 & 77 & 6,281 & 67 & 1,400 & 74 & 6,417 & 67 & 1,440 & 68 & 7,347 & 52 & 1,311 & 62 & 7,476 & 53 \\
\hline Other & 462 & 23 & 3,145 & 33 & 485 & 26 & 3,122 & 33 & 691 & 32 & 6,683 & 48 & 799 & 38 & 6,575 & 47 \\
\hline \multicolumn{17}{|l|}{ Marital Status: } \\
\hline Never married & 1,271 & 64 & 4,734 & 50 & 1,165 & 62 & 4,840 & 51 & 1,202 & 56 & 6,203 & 44 & 1,523 & 55 & 6,252 & 44 \\
\hline Other & 727 & 36 & 4,692 & 50 & 720 & 38 & 4,699 & 49 & 929 & 44 & 7,827 & 56 & 957 & 45 & 7,799 & 56 \\
\hline \multicolumn{17}{|l|}{ Diagnosis: } \\
\hline Schizophrenia & 1,564 & 78 & 6,676 & 71 & 1,396 & 74 & 6,844 & 72 & 1,380 & 65 & 8,640 & 61 & 1,277 & 60 & 8,743 & 62 \\
\hline Paranoia \& Acute Psychosis & 106 & 5 & 613 & 6 & 115 & 6 & 604 & 6 & 178 & 8 & 1,260 & 9 & 204 & 10 & 1,234 & 9 \\
\hline Major affective disorder & 114 & 6 & 806 & 9 & 143 & 8 & 777 & 8 & 193 & 9 & 1,647 & 12 & 254 & 12 & 1,586 & 11 \\
\hline Dementia & 150 & 7 & 981 & 10 & 171 & 9 & 960 & 10 & 261 & 12 & 1,778 & 13 & 264 & 13 & 1,772 & 13 \\
\hline Other & 64 & 4 & 350 & 4 & 59 & 3 & 355 & 4 & 119 & 6 & 705 & 5 & 111 & 5 & 716 & 5 \\
\hline
\end{tabular}

"Lowest SEIFA score for the areas in which the cohort members were in residence.

Table 4 reports the results of Logistic regression models that adjust for these differences in considering whether the CTOexperience was associated with initial perpetration and/or victimization risk after giving explanatory priority to potential confounding-risk-factors. The first set of models in Table 4, Model 1 , were statistically significant (when "initial-perpetration" was the dependent variable and all control variables were included: $\mathrm{N}=26,015, \chi^{2}=4737.18, \mathrm{df}=46,25,969, \mathrm{p}<.001$; similarly, when "initial-victimization" was the dependent variable: $\mathrm{N}=26,015$, $\chi^{2}=3162.98, \mathrm{df}=46,25,969, \mathrm{p}<.001$ ); and, CTO-placement was associated with reduced initial-perpetration and victimization risks. As the additional confounding-risk-factors were taken into account in stepwise fashion in Model 1, the association of reduced initial-perpetration-risk with CTO-assignment increased from $13 \%$ $(\operatorname{Exp}(\mathrm{B})=.87, \mathrm{CI} 95 \%=.80-.95, \mathrm{p}=.002)$, to $17 \%(\operatorname{Exp}(\mathrm{B})=.83, \mathrm{CI}$ $95 \%=.76-.91, \mathrm{p}<.001)$. For initial-victimization-risk, from $9 \%(\operatorname{Exp}$ $(\mathrm{B})=.91, \mathrm{CI} 95 \%=.84-.99, \mathrm{p}=.040)$, to $11 \%(\operatorname{Exp}(\mathrm{B})=.89, \mathrm{CI}$ $95 \%=.81-.97, \mathrm{p}=.011$ ) (See Table 4$)$. When the final fully controlled models were rerun without patients diagnosed with "dementia and other nervous system disorders", associations changed little: reduced initial-perpetration-risk was $15 \%(\operatorname{Exp}(B)=.85, \quad \mathrm{CI}$ $95 \%=.77-.94, \mathrm{p}=.002)$, initial-victimization-risk was $10 \%$ (Exp (B) $=.90$, CI 95\% $=.82-.99, \mathrm{p}=.028)$.

A small but significant association attributable to each community-treatment-day was found for initial-perpetrationrisk $(\operatorname{Exp}(B)=0.993, C I 95 \%=.990-.995, \mathrm{p}<.001)$, and initialvictimization-risk $(\operatorname{Exp}(B)=0.998$, CI 95\%=.996-.999, $\mathrm{p}<.040)$ (see Table 4, Model 1, models inclusive of all control variables). Given the small association effect for each community-treatment-day, the models were re-estimated evaluating the effect of "ten-community-treatment-days". During each community-careepisode, the significant interaction between ten-communitytreatment-days and CTO-placement was associated with a $3.4 \%$ reduced-initial-perpetration-risk for each additional ten- community-treatment-days $(\operatorname{Exp}(B)=.966$, CI $95 \%=.943-.988$; $\mathrm{p}=.003$ ), but not significantly related initial-victimization-risk.

In addition, when the two potential measures of intensity of community service were inserted into Model 1 (containing all control variables) for both initial perpetrations and victimizations, the "number of community-treatment-days per thirtydays" of a community-care-episode was associated with an increase in initial-perpetration risk $(\operatorname{Exp}(B)=1.010, \quad C I$ $95 \%=1.006-1.015, \mathrm{p}<.001)$ and not significantly associated with initial-victimization risk. The "number of contact hours per community-treatment-day" was not significantly associated with initial-perpetration-risk but was associated with a slight increase in initial-victimization-risk $(\operatorname{Exp}(B)=1.004$, $95 \%=1.000-1.008, \mathrm{p}=.042$ ).

The second set of Logistic regression models, Model 2, in Table 4 show the results of regressing exposure to at least one "СTOinitiated re-hospitalization" on initial-perpetration and initialvictimization. All the models were statistically significant. In the fully controlled models, when initial-perpetration was the dependent variable: $\mathrm{N}=26,015, \chi^{2}=4728.59, \mathrm{df}=46,25,969$, $\mathrm{p}<.001$; when initial-victimization was the dependent variable: $\mathrm{N}=26,015, \chi^{2}=3166.44, \mathrm{df}=46,25,969, \mathrm{p}<.001$. In these models, exposure to at least one "CTO-initiated re-hospitalization" was associated with a 13\% reduced-risk in initial-perpetration (Exp (b) $=.87, \mathrm{Cl} 95 \%=.77-.97, \mathrm{p}=.016)$ and a $17 \%$ reduced-risk in initialvictimization $(\operatorname{Exp}(\mathrm{b})=.83, \mathrm{CI} 95 \%=.74-.93, \mathrm{p}=.002)$ after taking into account all controls. When these models are re-run without patients with "dementia and other nervous system disorders", the associations with reduced-risk-estimates changed little. For exposure to at least one "CTO-initiated re-hospitalization" the association with reduced-risk in initial-perpetration remained $13 \%$ $(\operatorname{Exp}(b)=.87, C I 95 \%=.77-.98, p=.023)$, and was $15 \%$ for initialvictimization-risk $(\operatorname{Exp}(\mathrm{b})=.85, \mathrm{CI} 95 \%=.75-.95, \mathrm{p}=.006)$ after taking into account all controls. 
Table 4

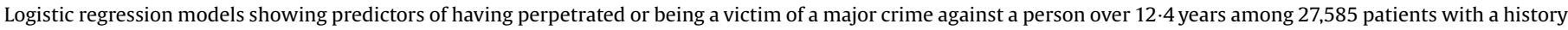
of psychiatric hospitalizationc.

\begin{tabular}{|c|c|c|c|c|c|c|c|c|c|c|c|}
\hline \multirow{2}{*}{\multicolumn{2}{|c|}{$\begin{array}{l}\text { Criterion Variable } \\
\text { Criterion Variable }\end{array}$}} & \multicolumn{5}{|c|}{$\begin{array}{l}\text { Perpetrated at least one crime } \\
\text { against a person }^{\text {a }}\end{array}$} & \multicolumn{5}{|c|}{$\begin{array}{l}\text { Victim of at least one crime against } \\
\text { a person }{ }^{b}\end{array}$} \\
\hline & & \multirow[t]{2}{*}{$b^{d}$} & \multirow[t]{2}{*}{ SE } & \multirow[t]{2}{*}{$\mathrm{P}$} & \multirow[t]{2}{*}{$\begin{array}{l}\text { Exp } \\
(b)^{\mathrm{e}}\end{array}$} & \multirow[t]{2}{*}{$95 \% \mathrm{CI}$} & \multirow[t]{2}{*}{$b^{d}$} & \multirow[t]{2}{*}{ SE } & \multirow[t]{2}{*}{$\mathrm{P}$} & \multirow[t]{2}{*}{$\begin{array}{l}\operatorname{Exp} \\
(b)^{\mathrm{e}}\end{array}$} & \multirow[t]{2}{*}{$95 \% \mathrm{CI}$} \\
\hline $\begin{array}{l}\text { Model 1: "CTO" and "Treatment Day } \\
\text { stepwise control for: ** }\end{array}$ & ect" variables with & & & & & & & & & & \\
\hline \multirow[t]{2}{*}{ Structural \& Bias Indicators ${ }^{* *}$} & СТO & -.138 & .04 & .002 & .871 & $.799-.949$ & -.091 & .04 & .040 & .913 & $.837-.996$ \\
\hline & $\begin{array}{l}\text { Treatment days per community care } \\
\text { episode }\end{array}$ & -.006 & .00 & $<.001$ & .994 & $.991-.997$ & -.002 & .00 & .083 & .998 & $\begin{array}{l}.996- \\
1.000\end{array}$ \\
\hline \multirow{2}{*}{$\begin{array}{l}\text { Plus Demographic, Diagnostic, and } \\
\text { Service Indicators }\end{array}$} & CTO & -.166 & .05 & $<.001$ & .847 & $.772-.930$ & -.110 & .05 & .017 & .896 & $.818-.981$ \\
\hline & $\begin{array}{l}\text { Treatment days per community care } \\
\text { episode }\end{array}$ & -.008 & .00 & $<.001$ & .992 & $.989-.995$ & -.003 & .00 & .013 & .997 & $.995-.999$ \\
\hline \multirow{2}{*}{$\begin{array}{l}\text { Plus Behavioral and Social } \\
\text { Circumstance Indicators }^{* *}\end{array}$} & СTO & -.183 & .05 & $<.001$ & .833 & $.757-.915$ & -.120 & .05 & .011 & .887 & $.809-.972$ \\
\hline & $\begin{array}{l}\text { Treatment days per community care } \\
\text { episode }\end{array}$ & -.008 & .00 & $<.001$ & .993 & $.990-.995$ & -.002 & .00 & .040 & .998 & $.996-.999$ \\
\hline \multicolumn{12}{|l|}{$\begin{array}{l}\text { Model 2: “CTO-initiated re- } \\
\text { hospitalization" and "Treatment Day } \\
\text { Effect" Variables with } \\
\text { stepwise control for: }{ }^{* *}\end{array}$} \\
\hline \multirow[t]{2}{*}{ Structural \& Bias Control Indicators ${ }^{* *}$} & CTO-initiated-re-hospitalization & .063 & .05 & .228 & 1.065 & $\begin{array}{l}.961- \\
1.179\end{array}$ & .007 & .05 & .899 & 1.007 & $.907-1.118$ \\
\hline & $\begin{array}{l}\text { Treatment days per community care } \\
\text { episode }\end{array}$ & -.006 & .00 & $<.001$ & .994 & $.991-.997$ & -.002 & .00 & .085 & .998 & $.996-1.00$ \\
\hline \multirow[t]{2}{*}{$\begin{array}{l}\text { Plus Demographic, Diagnostic, and } \\
\text { Service Indicators }\end{array}$} & CTO-initiated-re-hospitalization & -.066 & .06 & .235 & .936 & $\begin{array}{l}.839- \\
1.044\end{array}$ & -.089 & .06 & .110 & .914 & $\begin{array}{l}.820- \\
1.020\end{array}$ \\
\hline & $\begin{array}{l}\text { Treatment days per community care } \\
\text { episode }\end{array}$ & -.008 & .00 & $<.001$ & .992 & $.989-.995$ & -.003 & .00 & .015 & .997 & $.995-.999$ \\
\hline \multirow{2}{*}{$\begin{array}{l}\text { Plus Behavioral and Social } \\
\text { Circumstance Indicators }^{* *}\end{array}$} & CTO-initiated-re-hospitalization & -.141 & .06 & .016 & .868 & $.774-.974$ & -.183 & .06 & .002 & .833 & $.743-.933$ \\
\hline & $\begin{array}{l}\text { Treatment days per community care } \\
\text { episode }\end{array}$ & -.007 & .00 & $<.001$ & .993 & $.990-.996$ & -.002 & .00 & .047 & .998 & $.996-.999$ \\
\hline
\end{tabular}

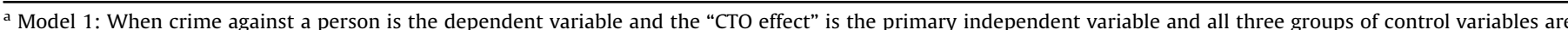

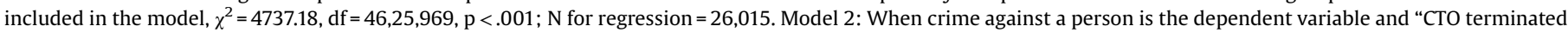

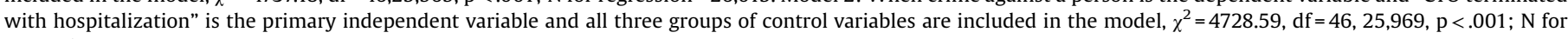
regression $=26,015$.

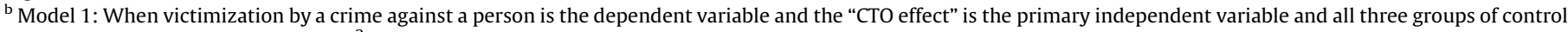
variables are included in the model, $\chi^{2}=3162.98, \mathrm{df}=46,25,969, \mathrm{p}<.001 ; \mathrm{N}$ for regression $=26,015$.

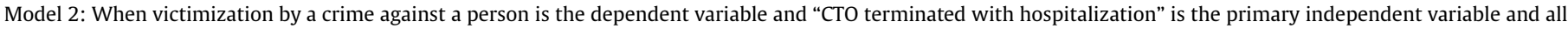
three groups of control variables are included in the model, $\chi^{2}=3166.44, \mathrm{df}=46,25,969, \mathrm{p}<.001 ; \mathrm{N}$ for regression $=26,015$.

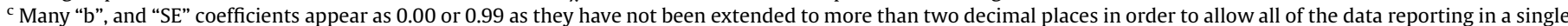
table. The zeros 0.00 or 0.99 refer to small and sometimes significant effects of the adjustment variables.

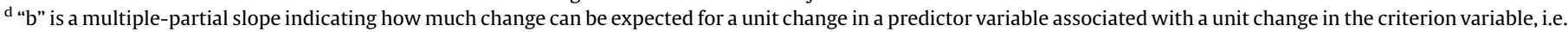
after all other predictors have been given causal priority in explaining the change.

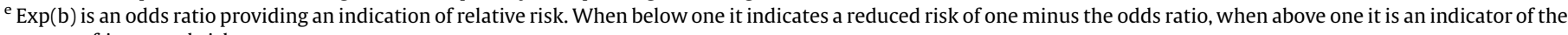
percent of increased risk.

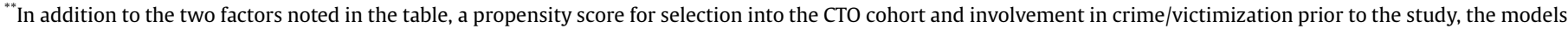
adjust for three groups of control variables by adding the additional controls to the models in steps:

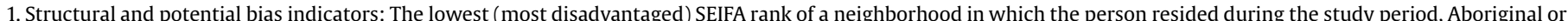

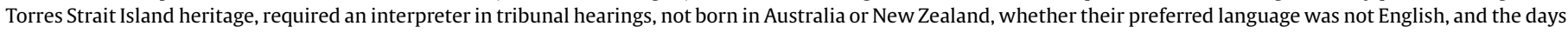
spent in prison during the study period.

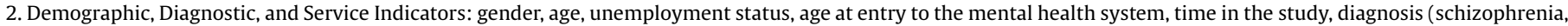
affective disorder, dementia, paranoia), duration of inpatient care, episode start year, $<11^{\text {th }}$ grade education.

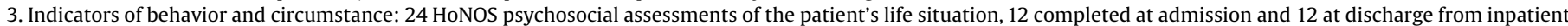
care over the study period.

There were 4129 individuals with one or more perpetrations ( $\mathrm{M}=3.6 \pm 4.3$, Median $=2.0$, Range 58), and 3995 with one or more victimizations ( $M=1.75 \pm 1.47$, Median 1.0, Range $=18$ ). Proportionally the groups did not differ significantly on either criterion. In fully controlled models, when repeat-perpetration is the dependent variable and CTO-supervision is the primary independent variable, model statistics are: $\mathrm{N}=3,973, \chi^{2}=743.62, \mathrm{df}=46,3927$, $\mathrm{p}<.001$ and CTO-use was associated with a $22 \%$ reduced-risk in repeat-perpetrations $(\operatorname{Exp}(B)=.78, C I 95 \%=.66-.93, \mathrm{p}=.006)$. CTOinitiated re-hospitalization was only marginally significant in association with repeat-perpetrations $(\operatorname{Exp}(B)=.82$, CI $95 \%=.66-$ $1.00, \mathrm{p}=.054)$. When repeat-victimization is the dependent variable, only CTO-initiated re-hospitalization was significant as a primary independent variable. Model statistics are: $\mathrm{N}=3859$, $\chi^{2}=249.73, \mathrm{df}=46,3813, \mathrm{p}<.001$. CTO-initiated return to hospital was associated with a $22 \%$ reduced-risk in repeat-victimization $(\operatorname{Exp}(B)=.78, C I 95 \%=.66-.95, \mathrm{p}=.017)$.

\section{Discussion}

During the 12.4-year study-period the crude-perpetrationrates per 100,000 , respectively for the CTO and Non-CTO cohorts, were 7.5 and 5.6-times higher than for Victoria's citizens. Crudevictimization-rates were respectively 3.7 and 3-times higher (Table 2). The higher rates seem to confirm that hospitalizedpsychiatric-patients posed a greater safety-threat to others and to themselves, exclusive of self-harm, than do members of the general-public. 
The numbers of hospitalized patients that engaged in at least one perpetration or experienced a victimization was proportionally greater in the CTO vs the Non-CTO-cohort (Table 3 ). From these statistics, it would appear that CTOs are not likely to address the frequency of crime even within the hospitalized population. These statistics are, however, crude figures that do not take into account the fact that the CTO-cohort was characterized by more risk-factors associated with violent crime and victimization than the non-CTOcohort. The CTO-cohort included younger unemployed males living in more socially disadvantaged neighborhoods. Its HoNOS severity-profiles appeared to validate a need for treatment exceeding that of the non-CTO-cohort, particularly in areas of potentially dangerous behavior [27]. The Logistic models enabled adjustment for between group differences (Table 4). They controlled for prior crimes and gave explanatory priority to alternative-risk-factors over the CTO in assessing perpetration and victimization risk. After these potentially confounding factors were taken into account, the CTO was associated with a $17 \%$ reduced-risk in initial-perpetration and an $11 \%$ reduced-risk in initial-victimization. CTO-supervision was also associated with a reduced-risk of $22 \%$ in repeat-perpetration. These results echo findings of dramatic decreases in police contacts and violent behavior associated with OCC-implementation in New York [21,22].

Studies using re-hospitalization as their primary outcome measures, including the three randomized OCC-studies [46-48], unfortunately, conflate their outcome-measure with their intervention-measure-i.e., providing supervision enabling timelyreturn to hospital when new symptoms threaten to have dangerous consequences. This conflated dependent variable makes it difficult to determine whether the results of these trials are positive or negative [49]. The findings herein support CTOs' protective role of providing needed community-based treatment and providing needed-treatment via re-hospitalization. Community-treatment-days, in the context of Victoria's reduced community-service-commitments [27], when compared to rehospitalization, appeared to play a relatively smaller part in association with reduced perpetration and victimization-risk. CTO-initiated re-hospitalization was associated with a 13\% reduced-risk in initial-perpetration, a $17 \%$ reduced-risk in initialvictimization, and a $22 \%$ reduced-risk in repeat-victimization. There was no significant relationship between initial-victimization-risk for every "ten-community-treatment-days" associated with CTO-placement, though there was an associated $3.4 \%$ reduced-risk in initial perpetration, amounting to a $9.0 \%$ reduced-risk in initial-perpetration for the average CTO-patient who received 26.6 community-treatment-days per communitycare-episode vs. the 16.6 days in the non-CTO-group.

Victoria's experience seems to follow an international deinstitutionalization-dynamic where mental health systems are reorganized around general-hospital-integrated-care [50]. The mandated objectives of OCC, protecting safety of self and others from the behavioral consequences of severe mental illness, are displaced by the goal of "prevention of hospitalization", stigmatizing hospital-use. This empowers factions opposing hospitalization based on human rights concerns and those groups committed to cost-savings in mental health services to cut beds [51]. Reduced availability of generalhospital-beds creates pressure for earlier discharge to accommodate new acute patients [52]. In the absence of strong Assertive Community Treatment or equivalent-case-management, hastened discharge leads to a high probability of readmission for symptoms associated with the original hospital-stay [53]. The "revolving-door" effect is exacerbated in managed-care-systems where hospitals are paid a fixed fee per admission-profiting when admissions are short and re-hospitalization is billable as a new encounter. CTOs, in the absence of adequate community-care, carry out their mandate to enable the provision of needed-treatment: crisis-returns to hospital, the default requirement in the absence of adequate-community-treatment [49]. Critics, conflating outcome and treatment intervention, misrepresent this intervention as CTO's failure to "prevent hospitalization", making no mention of inadequate community-based treatment, cutbacks in such service associated with concentrating care in the general hospital complex, the financing incentives for readmissions [54], and the dire behavioral risks to long-term recovery potentially avoided with CTOsupervision. It is time to add greater legitimacy to both the need for expanded community and hospital-based resources for neededtreatment as well as less restrictive alternatives to the criminal justice system, which is the next stop for many denied access to inpatient care when they need it [55].

Herein we have addressed only two of the many ways OCC may contribute to providing needed treatment to protect health and safety. There are many threats to health and safety where the evaluation of the role of OCC requires different outcome measures. In order to improve and better understand OCC we need future research efforts focused on other statutorily justified objectives that are evaluated with behavioral outcomes that would indicate a success or failure of OCC in meeting these objectives.

The study's limitations are inherent in issues that impede direct generalization of crime data to other nations [56], its use of administrative and criminal justice data, and its correlational casecontrol methods. Though it may be possible that a return to hospital ending a CTO-episode was independently initiated, this was unlikely without the influence of the mental health team. While the correlational methods do not confirm causation these methods remain among the best available. In this area, a substantively valid clinical trial does not appear to be possible for ethical and legal reasons [57-59]. The adjustments or controls for confounding influences in the statistical models herein give all forty-six control factors explanatory priority over the explanation that the CTO is accounting for the study results. The models therefore provide a conservative estimate of the CTO association. Finally, the study results were robust in the face of a challenge to their diagnostic generalizability.

\section{Author contributions}

All authors have contributed to the conception and design, or analysis and interpretation of data, the drafting and revisions of the article, and its critically important and intellectual content. All have given approval of the manuscript submission.

\section{Conflicts of interest}

Authors Have No Conflicts Of Interest Associated With This Study.

\section{Appendix A. Supplementary data}

Supplementary data associated with this article can be found, in the online version, at https://doi.org/10.1016/j.eurpsy.2018.12.001.

\section{References}

[1] Bazelon center for mental health law. Washington D.C: Involuntary outpatient commitment: summary of state statutes. Bazelon Center for Mental Health Law; 2004. http://www.bazelon.org.

[2] Dennis H., 647 N.W.2d (Wis,2002) p. 863.

[3] Glover-Thomas NR. Reconstructing mental health law and policy. London: Lexis Nexis Butterworths Tolley; 2002.

[4] U.S. Civil Commitment Laws by State. Downloaded 30 December, 2017 from http://www.treatmentadvocacycenter.org/browse-by-state; Victoria Mental Health Act 1986, No. 59 of 1986, Version No. 098;Version incorporating amendments as at 24 August 2010. http://www.legislation.vic.gov.au/Domino/ Web_Notes/LDMS/LTObject_Store/LTObjSt5.nsf/ DDE300B846EED9C7CA257616000A3571/ A7D40FD608D61455CA2577890007FEB8/\$FILE/86-59a098.pdf Mental Health 
Act 1996 (Tas) s 24. http://www.dhhs.tas.gov.au/mentalhealth/ mental_health_act/mental_health_act_2013_new_mental_health_act/ information_for_consumers_carers_and_the_community_sector/fact_sheets/ treatment orders under the mental health act 2013 Mental Health Act 2000 (NSW) s 14(1)(b). Mental Health Act 1998 (WA) s 26(2). Mental Health Act 2000 (Qld) s 14(1)(f). ttps://www.legislation.qld.gov.au/view/pdf/200207-19/act-2000-016 UK Mental Health Act 2007-8, www.legislation.gov.uk/ ukpga/2007/12/pdfs/ukpga_20070012 en.pdf;.

[5] U.K. Parliament Health Committee. Post-legislative scrutiny of the mental health act 2007 - health committee contents, sec 5 supervised Community treatment, \#80. 2013. . Downloaded 24/2/18 https://publications.parliament. uk/pa/cm201314/cmselect/cmhealth/584/58408.htm.

[6] Treatment Advocacy Center. Risk factors for violence in serious mental illness. Washington D.C, USA: Treatment Advocacy Center: Office of Research and Public Affairs; 2016. . downloaded 11/10 18 https://www. treatmentadvocacycenter.org/storage/documents/backgrounders/smi-andrisks-for-violence.pdf).

[7] Teplin LA, McClelland GM, Abram KM, Weiner DA. crime victimization in adults with severe mental illness: comparison with the national crime victimization survey. Arch Gen Psychiatry 2005;62(8)911-21 2005.

[8] Hiday VA, Swartz MS, Swanson JW, Borum R, Wagner HR. Criminal victimization of persons with severe mental illness. Psychiatr Serv 1999;50:62-8 [PubMed: 9890581].

[9] Marley JA, Buila S. Crimes against people with mental illness: types, perpetrators, and influencing factors. Soc Work 2001;46:115-24 [PubMed: $11329641]$.

[10] Goodman LA, Rosenberg SD, Mueser KT, Drake RE. Physical and sexual assault history in women with serious mental illness: prevalence, correlates, treatment, and future research directions. Schizophrenia Bull 1997;23:685-96 [PubMed: 9366004].

[11] Darves-Bornoz JM, Lemperiere T, Degiovanni A, Gaillard P. Sexual victimization in women with schizophrenia and bipolar disorder. Soc Psychiatry Psychiatric Epidemiol 1995;30:78-84 [PubMed: 7754420].

[12] Hiday VA. The social context of mental illness and violence. J Health Soc Behav 1995;36:122-37 [PubMed: 9113138].

[13] Sells DJ, Rowe M, Fisk D, Davidson L. Violent victimization of persons with cooccurring psychiatric and substance use disorders. Psychiatric Serv 2003;54:1253-7 [PubMed: 12954942].

[14] Logan TK, Walker R, Cole J, Leukefeld C. Victimization and substance abuse among women: contributing factors, interventions, and implications. Rev Gen Psychol. 2002;6:325-97.

[15] Miethe TD, McDowall D. Contextual effects in models of criminal victimization. Soc Forces 1993;71:741-61.

[16] Cunradi CB, Caetano R, Clark CL, Schafer J. Alcohol-related problems and intimate partner violence among white, black, and Hispanic couples in the U.S. Alcohol Clin Exp Res 1999;23:1492-501 [PubMed: 10512315].

[17] Cunradi CB, Caetano R, Schafer J. Socioeconomic predictors of intimate partner violence among white, black, and Hispanic couples in the United States. J Fam Violence 2002;17:377-89.

[18] Silver E. Mental disorder and violent victimization: the mediating role of involvement in conflicted social relationships. Criminology 2002;40:191-212.

[19] RachBeisel J, Scott J, Dixon L. Co-occurring severe mental illness and substance use disorders: a review of recent research. Psychiatric Serv 1999;50:1427-34 [PubMed: 10543851].

[20] Hiday VA, Swartz MS, Swanson JW, Borum R, Wagner HR. Impact of outpatient commitment on victimization of people with severe mental illness. Am J Psychiatry 2002;159:1403-11.

[21] Link BG, Epperson MW, Perron BE, Castille DM, Yang LH. Arrest outcomes associated with outpatient commitment in New York State. Psychiatric Serv 2011:62:504-8.

[22] Phelan JC, Sinkewicz M, Castille DM, Huz S, Link BG. Effectiveness and outcomes of assisted outpatient treatment in New York State. Psychiatric Serv 2010;61:137-43.

[23] Segal SP, Hayes SL, Rimes L. The utility of outpatient commitment: II. Mortality risk, protecting health, safety, and quality of life. Psychiatric Serv 2017;68:1255-61.

[24] Kisely S, Preston N, Xiao J, Lawrence D, Louise S, Crowe E. Reducing all-cause mortality among patients with psychiatric disorders: a population-based study. Can Med Assoc J 2012;185(1):E50-6.

[25] Segal S, Burgess P. Effect of conditional release from hospitalization on mortality-risk. Psychiatric Serv 2006;57:1607-13.

[26] Lake v. Cameron, 364 F.2d 657 (1966).

[27] Segal SP, Hayes SL, Rimes L. The utility of outpatient commitment: I. A need for treatment and a least restrictive alternative to psychiatric hospitalization. Psychiatric Serv 2017;68:1247-54.

[28] Segal SP. Civil commitment law, mental health services, and US homicide rates. Soc Psychiatry Psychiatric Epidemiol 2012;47:1449-58.

[29] Large M, Nielssen O. Evidence for a relationship between the duration of untreated psychosis and the proportion of psychotic homicides prior to treatment. Soc Psychiatry Psychiatric Epidemiol 2008;43:37-44.

[30] Gerrand V. Transforming mental health services from 1993 to 1998 in Victoria, Australia: a case study of policy implementation. University of Melbourne: Department of Political Science; 2005.
[31] Victoria Police. About Victoria Police: Victoria Police Crime Statistics. State of Victoria. Availablefrom: http://www.police.vic.gov.au/content.asp? Document_ID=782.

[32] Australian Bureau of Statistics. Technical paper: socio-economic indexes for areas (SEIFA). Commonwealth of Australia; 2011.

[33] Burgess P, Coombs T, Clarke A, et al. Achievements in mental health outcome measurement in Australia: reflections on progress made by the Australian mental health outcomes and classification network (AMHOCN). Int J Mental Health Syst 2012;6:4.

[34] Victoria DHHS. Service contacts in CMI/ODS: program management circular. Victoria DHHS, Melbourne Australia; 2017. . Downloaded 16 November, 2018 https://www2.health.vic.gov.au/mental-health/research-and-reporting/ reporting-requirements-for-clinical-mental-health-services/service-contacts.

[35] Tansella M, Micciolo R, Biggeri A, Bisoffi G, Balestrieri M. Episodes of care fo first-ever psychiatric patients. A long-term case-register evaluation in a mainly urban area. Br J Psychiatry 1995;167:220-7.

[36] Police V. offences recorded by offence category, offence code, description and statutory reference, 2004/05-2013/14. Victoria Police; 2015 Available from: https://www.police.vic.gov.au/retrievemedia.asp?Media . . .

[37] Australian Bureau of Statistics. TABLE 4. Estimated resident population, States and Territories. Australian bureau of statistics.

[38] Sampson RJ, Raudenbush SW, Earls F. Neighborhoods and violent crime: a multilevel study of collective efficacy. Science 1997;277:918-24.

[39] Eagar K, Buckingham B, Coombs T, et al. Victorian outcome measurement strategy Resource manual: AMHOCN health of the nation outcome scales (HoNOS): glossary. Parramata, New South Wales, Australian Mental Health Outcomes and Classification Network; 2000.

[40] Phelan M, Slade M, Thornicroft G, Dunn G, Holloway F, Wykes T, et al. the camberwell assessment of need: the validity and reliability of an instrument to assess the needs of people with severe mental illness. Br J Psychiatry 1995; 167:589-95.

[41] Wing J, Beevor A, Curtis R, Park S, Hadden S, Burns A. Health of the nation outcome scales (HoNOS). Research and development. Br J Psychiatry 1998; $172: 11-8$.

[42] Royal College of Psychiatrists. Frequently asked questions about HoNOS. downloaded 2015. London: Royal College of Psychiatrists; 2015. http://www. rcpsych.ac.uk/traininpsychiatry/training/honos/generalinformation/faq. aspx\#scalescover.

[43] Pirkis J, Burgess P, Coombs T, et al. Routine measurement of outcomes in Australia's public sector mental health services. Aust N Z Health Policy 2005;2:8.

[44] Burgess P, Trauer T, Coombs T, et al. What does "clinical significance"mean in the context of the health of the nation outcome scales? Aust Psychiatry 2009; 17:141-8.

[45] IBM Corp. IBM SPSS statistics for windows, version 24.0. Armonk, NY: IBM Corp; 2017.

[46] Steadman HJ, Gounis K, Dennis D, Hopper K, Roche B, Swartz M, et al. Assessing the New York City involuntary outpatient commitment pilot program. Psychiatric Serv 2001;52:330-6.

[47] Swartz M, Swanson JD, et al. Can involuntary outpatient commitment reduce hospital recidivism? Findings from a randomized trial with severely mentally ill individuals. Am J Psychiatry 1999;156:1968-75.

[48] Burns T, Rugkåsa J, Molodynski A, Dawson J, Yeeles K, Vazquez-Montes M, et al Community treatment orders for patients with psychosis (OCTET): a randomised controlled trial. Lancet 2013;381:1627-33.

[49] Segal SP. Assessment of outpatient commitment in randomised trials. LancetPsychiatry 2017;4(12):e26-8.

[50] Segal SP, Jacobs L, Deinstitutionalization. Encyclopedia of social work. 21th edition Co- published by the NASW Press and Oxford University Press; 2013.

[51] Slade EP, Goldman HH. The dynamics of psychiatric bed use in genera hospitals. Adm Policy Ment Health 2015;42(March (2)):139-46, doi:http://dx. doi.org/10.1007/s10488-014-0554-4.

[52] Torrey EF, Fuller DA, Geller J, Jacobs C. No room at the Inn: trends and consequences of closing public psychiatric hospitals. Washington DC: Treatment Advocacy Center; 2012.

[53] Segal SP, Watson M, Akutsu P. Factors associated with involuntary return to a psychiatric emergency service within twelve months. Psychiatric Serv 1998;49 (9):1212-7.

[54] Patel G. Community treatment orders in Victoria: a clinico-ethical perspective. Aust Psychiatry 2008;16(5):340-3.

[55] Talbot D. Legal system's treatment of the mentally ill is truly criminal. Thursday, May 25, Sec D, pp. 1-2.. San Francisco Chronical; 2017. www.SFChronical.com.

[56] Farrington D, Langa P, Tonry M. Cross national studies in crime and justice. Bureau of justice statistics. Washington, DC: U.S. Department of Justice: Office of Justice Programs; 2004.

[57] O'Reilly R, Vingilis E. Are Randomized Control Trials the Best Method to Assess the Effectiveness of Community Treatment Orders? Administration and Policy in Mental Health and Mental Health Services Research. , doi:http://dx.doi.org/ 10.1007/s10488-017-0845-7 Online.

[58] Hawkins AJ. Realist evaluation and randomised controlled trials for testing program theory in complex social systems. Evaluation 2016;22:270-85.

[59] Mustafa FA. Notes on the use of randomised controlled trials to evaluate complex interventions: community treatment orders as an illustrative case. J Eval Clin Pract 2017;23:185-92. 